\title{
High-pressure hydrogen sulfide by diffusion quantum Monte Carlo
}

Sam Azadi, and Thomas D. Kühne

Citation: The Journal of Chemical Physics 146, 084503 (2017); doi: 10.1063/1.4976836

View online: https://doi.org/10.1063/1.4976836

View Table of Contents: http://aip.scitation.org/toc/jcp/146/8

Published by the American Institute of Physics

\section{Articles you may be interested in}

The metallization and superconductivity of dense hydrogen sulfide

The Journal of Chemical Physics 140, 174712 (2014); 10.1063/1.4874158

Synthesis and stability of hydrogen selenide compounds at high pressure

The Journal of Chemical Physics 147, 184303 (2017); 10.1063/1.5004242

Perspective: Role of structure prediction in materials discovery and design APL Materials 4, 053210 (2016); 10.1063/1.4949361

Revisiting the hydration structure of aqueous $\mathrm{Na}^{+}$

The Journal of Chemical Physics 146, 084504 (2017); 10.1063/1.4975608

Coupled electron-ion Monte Carlo simulation of hydrogen molecular crystals

The Journal of Chemical Physics 148, 102314 (2018); 10.1063/1.5001387

Perspective: Crystal structure prediction at high pressures

The Journal of Chemical Physics 140, 040901 (2014); 10.1063/1.4861966

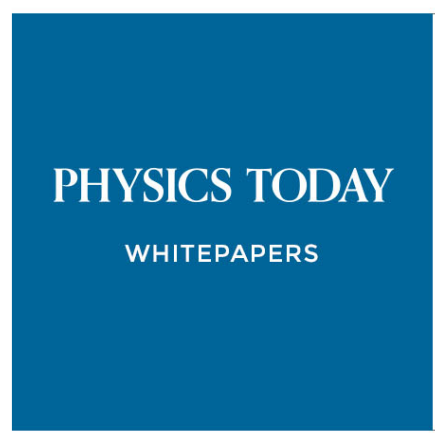




\title{
High-pressure hydrogen sulfide by diffusion quantum Monte Carlo
}

\author{
Sam Azadi ${ }^{1, a)}$ and Thomas D. Kühne ${ }^{2}$ \\ ${ }^{1}$ Department of Materials Science, Royal School of Mines, Thomas Young Center, London Centre \\ for Nanotechnology, Imperial College London, London SW7 2AZ, United Kingdom \\ ${ }^{2}$ Dynamics of Condensed Matter, Department of Chemistry, University of Paderborn, Warburger Strasse 100 , \\ D-33098 Paderborn, Germany and Paderborn Center for Parallel Computing and Institute for Lightweight \\ Design with Hybrid Systems, University of Paderborn, Warburger Strasse 100, D-33098 Paderborn, Germany
}

(Received 29 August 2016; accepted 6 February 2017; published online 27 February 2017)

\begin{abstract}
We revisit the enthalpy-pressure phase diagram of the various products from the different proposed decompositions of $\mathrm{H}_{2} \mathrm{~S}$ at pressures above $150 \mathrm{GPa}$ by means of accurate diffusion Monte Carlo simulations. Our results entail a revision of the ground-state enthalpy-pressure phase diagram. Specifically, we find that the $\mathrm{C} 2 / \mathrm{c} \mathrm{HS}_{2}$ structure is persistent up to $440 \mathrm{GPa}$ before undergoing a phase transition into the $\mathrm{C} 2 / \mathrm{m}$ phase. Contrary to density functional theory, our calculations suggest that the $\mathrm{C} 2 / \mathrm{m}$ phase of HS is more stable than the I $4_{1} /$ amd HS structure over the whole pressure range from 150 to $400 \mathrm{GPa}$. More importantly, we predict that the Im-3m phase is the most likely candidate for $\mathrm{H}_{3} \mathrm{~S}$, which is consistent with recent experimental x-ray diffraction measurements. Published by AIP Publishing. [http://dx.doi.org/10.1063/1.4976836]
\end{abstract}

\section{INTRODUCTION}

Back in 1968, Ashcroft predicted that according to the BCS theory, ${ }^{1}$ dense hydrogen would not only be metallic ${ }^{2}$ but more importantly also a high-temperature superconductor. ${ }^{3}$ Since recently it has been shown that dissociation is a necessary condition for the metallization of hydrogen, ${ }^{4,5}$ the necessary pressure to cause bandgap closure has remained impractically high, so that metallic hydrogen has only been realized at finitetemperature. ${ }^{6-10}$ Yet, an appealing way to circumvent the high pressures required to metallize hydrogen is to precompress it in hydrogen-rich systems, ${ }^{11,12}$ since, in general, the electronic density of states is high and the electron-phonon interactions are strong. ${ }^{13}$ In fact, pressurized hydrogen-rich materials have demonstrated to be rather promising candidates for high- $\mathrm{T}_{c}$ superconductivity. ${ }^{14-20}$

The possibility of high- $\mathrm{T}_{c}$ superconductivity of dense hydrogen sulfide was first predicted based on density functional theory (DFT) calculations. ${ }^{21,22}$ Experimentally, Drozdov and Eremets reported that at pressures around 200 GPa, dense $\mathrm{H}_{2} \mathrm{~S}$ becomes metallic and superconducting with a critical temperature $\left(T_{c}\right)$ of $203 \mathrm{~K},{ }^{23,24}$ which is well above the highest $\mathrm{T}_{c}$ of $133 \mathrm{~K}$ and $164 \mathrm{~K}$ that were achieved in cuprates ${ }^{25}$ at ambient ${ }^{26}$ and high pressures, ${ }^{27}$ respectively. Recent synchrotron $\mathrm{x}$-ray diffraction (XRD) measurements indicate that under these conditions $\mathrm{H}_{2} \mathrm{~S}$ consists of two major phases, elemental sulfur of the $\beta$-Po structure ${ }^{28}$ as well as body-centered cubic (bcc) $\mathrm{H}_{3} \mathrm{~S} .{ }^{29}$ The latter immediately suggests a decomposition via the $3 \mathrm{H}_{2} \mathrm{~S} \rightarrow 2 \mathrm{H}_{3} \mathrm{~S}+\mathrm{S},{ }^{29}$ in agreement with previous calculations. ${ }^{22,30-32}$ The crystal structure of the superconducting phase of $\mathrm{H}_{3} \mathrm{~S}$ was theoretically predicted to be the body-centered cubic (bcc) R3m and Im-3m phases at pressures above $110 \mathrm{GPa}$ and $180 \mathrm{GPa}$, respectively. ${ }^{22}$ Recent Raman

a)s.azadi@imperial.ac.uk spectroscopy and synchrotron x-ray diffraction experiments show that $\mathrm{H}_{2} \mathrm{~S}$ becomes unstable with respect to either $\mathrm{R} 3 \mathrm{~m}$ or $\operatorname{Im} 3 \mathrm{~m}$ and the $\mathrm{Cccm}$ phase of $\mathrm{H}_{3} \mathrm{~S}^{33,34}$

Numerous theoretical studies of the $\mathrm{Im}-3 \mathrm{~m} \mathrm{H}_{3} \mathrm{~S}$ structure consistently predict $T_{c}>200 \mathrm{~K},,^{30-32,35-38}$ in agreement with experiment. ${ }^{23,24}$ However, experimental XRD measurements are unable to distinguish between the two theoretically predicted bec phases of $\mathrm{H}_{3} \mathrm{~S}$ since the positions of the sulfur atoms are almost identical and the only difference is in the positions of the weakly scattering hydrogen atoms. Yet, the inclusion of zero-point energy (ZPE) leads to an alternative decomposition, namely, $5 \mathrm{H}_{2} \mathrm{~S} \rightarrow 3 \mathrm{H}_{3} \mathrm{~S}+\mathrm{HS}_{2}$, where both $\mathrm{H}_{3} \mathrm{~S}$ and $\mathrm{HS}_{2}$ are metallic and exhibit superconductive behavior. ${ }^{35}$

The transition from the $\mathrm{H}_{3} \mathrm{~S} R 3 \mathrm{~m}$ phase to the $\mathrm{H}_{3} \mathrm{~S} \mathrm{Im}-3 \mathrm{~m}$ happens at about $175 \mathrm{GPa}$ within DFT calculations in which the generalized gradient approximation of Perdew-BurkeErnzerhof (PBE) ${ }^{39}$ is employed. ${ }^{22}$ Using the local-densityapproximation (LDA) exchange-correlation functional gives a transition pressure of $145 \mathrm{GPa}$ for the same structural transformation. ${ }^{32,38}$ In the $\mathrm{R} 3 \mathrm{~m}$ phase, the $\mathrm{H}$ atoms sit in an off-center position forming a covalent bond with $\mathrm{S}$ atom and a hydrogen bond with another S, while in the Im-3m phase, the $\mathrm{H}$ atom is symmetrized and sits exactly at the same distance between the two $\mathrm{S}$ atoms. The ZPE modifies the pressure at which the R3m to $\mathrm{Im}-3 \mathrm{~m}$ transition occurs. It has been predicted that in $\mathrm{H}_{3} \mathrm{~S}$ there is a quantum hydrogen bond symmetrization, similar to the situation in ice $\mathrm{X}^{38}$

However, nearly all of the recent calculations on hydrogen-rich systems are based on the single-particle mean-field theories such as density-functional theory (DFT). ${ }^{12,15-22,30-32,35-38,40-46}$ Even though formally exact, the exact exchange and correlation (XC) functional is unknown from the outset and needs to be approximated, which affects both the relative stabilities of the different crystal structures. Indeed, for dense hydrogen-rich materials, a significant dependence on the particular of XC functional was established. ${ }^{4,47-49}$ 
Therefore, in this paper we revisit the stability of the individual products originating from the various proposed decompositions of $\mathrm{H}_{2} \mathrm{~S}$ for pressures above $150 \mathrm{GPa}$ by means of highly accurate diffusion Monte Carlo (DMC) simulations. Using the DMC method, ${ }^{50,51}$ the electronic manybody Schrödinger equation is solved stochastically, which have yielded very accurate total energies for atoms, ${ }^{52,53}$ molecules, ${ }^{54-56}$ and crystals ${ }^{57-59}$ including hydrogen-rich materials at very high pressure. ${ }^{5,60-62}$

\section{COMPUTATIONAL DETAILS}

At first, all of the examined structures were determined by relaxing the internal parameters of each phase within DFT at fixed external pressure. The DFT calculations were all conducted within the pseudopotential and plane-wave approach using the CASTEP code. ${ }^{63}$ Specifically, ultrasoft pseudopotentials were employed together with an energy cutoff of $1000 \mathrm{eV} \cdot{ }^{64}$ For $\mathrm{S}$ element, 6 electrons of $3 s$ and $3 p$ orbitals are selected as the valence electrons. The exact XC functional was substituted by the generalized gradient approximation of Perdew-Burke-Ernzerhof (PBE). ${ }^{39}$ The same XC functional is employed to generate the ultrasoft pseudopotentials. The geometry and cell optimizations were conducted using the BFGS algorithm and a dense $16 \times 16 \times 16 \mathbf{k}$-point mesh to sample the Brillouin zone, while the nuclear forces and components of the stress tensor were converged to $0.01 \mathrm{eV} / \AA$ and $0.01 \mathrm{GPa}$, respectively. Our DFT-PBE results agree with previous simulations.

The CASINO code was used to perform fixed-node DMC simulations with a trial wave function of the Slater-Jastrow (SJ) form, ${ }^{65}$

$$
\Psi_{\mathrm{SJ}}(\mathbf{R})=\exp [J(\mathbf{R})] \operatorname{det}\left[\psi_{n}\left(\mathbf{r}_{i}^{\uparrow}\right)\right] \operatorname{det}\left[\psi_{n}\left(\mathbf{r}_{j}^{\downarrow}\right)\right],
$$

where $\mathbf{R}$ is a $3 N$-dimensional vector containing the positions of all $N$ electrons, $\mathbf{r}_{i}^{\uparrow}$ the position of the $i$ th spin-up electron, $\mathbf{r}_{j}^{\downarrow}$ the position of the $j$ th spin-down electron, $\exp [J(\mathbf{R})]$ a Jastrow factor, and $\operatorname{det}\left[\psi_{n}\left(\mathbf{r}_{i}^{\uparrow}\right)\right]$ and $\operatorname{det}\left[\psi_{n}\left(\mathbf{r}_{j}^{\downarrow}\right)\right]$ are Slater determinants made of spin-up and spin-down one-electron wave functions. These orbitals were obtained from PBE-DFT calculations performed with the CASTEP plane-wave code ${ }^{63}$ in conjunction with Trail-Needs Hartree-Fock pseudopotentials. ${ }^{66,67}$ For the purpose to approach the complete basis set limit, ${ }^{68}$ a large energy cutoff of $4000 \mathrm{eV}$ has been chosen. The resulting planewave orbitals were subsequently transformed into a localized "blip" polynomial basis. ${ }^{69}$

The Jastrow factor within Eq. (1) is a positive, symmetric, explicit function of interparticle distances. The employed Jastrow factor includes polynomial one-body electron-nucleus (1b), two-body electron-electron (2b), and three-body electron-electron-nucleus (3b) terms, as well as plane-wave expansions of the electron-electron separation known as $p$ terms. ${ }^{70}$ These $p$ terms build long-ranged correlations into the Jastrow factor and thus significantly improve the wave function and variational energy. We also investigated the effect of the inhomogenous backflow (BF) coordinate transformation on the VMC and DMC total energies. ${ }^{71}$ Our BF transformation includes electron-electron and electron-proton terms and is given by
TABLE I. Total energies of the $\mathrm{C} 2 / \mathrm{m}$ phase of $\mathrm{HS}_{2}$ at the DMC level of theory for four different pressures. The energies are given in eV/atom and are calculated for $N_{1}=48, N_{2}=96$, and $N_{3}=192$ particles in the unit cell, respectively. The extrapolated DMC energy at the infinite system size limit is denoted by $\mathrm{E}(\infty)$.

\begin{tabular}{lcccc}
\hline \hline $\mathrm{P}(\mathrm{GPa})$ & $\mathrm{E}\left(N_{1}\right)$ & $\mathrm{E}\left(N_{2}\right)$ & $\mathrm{E}\left(N_{3}\right)$ & $\mathrm{E}(\infty)$ \\
\hline 150 & $-187.6003(8)$ & $-187.3893(6)$ & $-187.2745(6)$ & $-187.169(2)$ \\
200 & $-186.9612(8)$ & $-186.7573(8)$ & $-186.6484(5)$ & $-186.546(2)$ \\
250 & $-186.3569(9)$ & $-186.1696(8)$ & $-186.0580(6)$ & $-185.964(2)$ \\
300 & $-185.7804(8)$ & $-185.6074(6)$ & $-185.4851(7)$ & $-185.399(2)$ \\
\hline \hline
\end{tabular}

$$
\mathbf{X}_{i}\left(\left\{\mathbf{r}_{j}\right\}\right)=\mathbf{r}_{i}+\boldsymbol{\xi}_{i}^{(e-e)}\left(\left\{\mathbf{r}_{j}\right\}\right)+\boldsymbol{\xi}_{i}^{(e-P)}\left(\left\{\mathbf{r}_{j}\right\}\right)
$$

where $\mathbf{X}_{i}\left(\left\{\mathbf{r}_{j}\right\}\right)$ is the transformed coordinate of electron $i$, which depends on the full configuration of the system $\left\{\mathbf{r}_{j}\right\}$. The vector functions $\boldsymbol{\xi}_{i}^{(e-e)}\left(\left\{\mathbf{r}_{j}\right\}\right)$ and $\boldsymbol{\xi}_{i}^{(e-P)}\left(\left\{\mathbf{r}_{j}\right\}\right)$ are the electron-electron and electron-proton backflow displacements of electron $i$. They are parameterized as

$$
\boldsymbol{\xi}_{i}^{(e-e)}\left(\left\{\mathbf{r}_{j}\right\}\right)=\sum_{j \neq i}^{N_{e}} \alpha_{i j}\left(r_{i j}\right) \mathbf{r}_{i j}
$$

and

$$
\xi_{i}^{(e-P)}\left(\left\{\mathbf{r}_{j}\right\}\right)=\sum_{I}^{N_{P}} \beta_{i I}\left(r_{i I}\right) \mathbf{r}_{i I},
$$

where $\alpha_{i j}\left(r_{i j}\right)$ and $\beta_{i I}\left(r_{i I}\right)$ are polynomial functions of electronelectron and electron-proton distances that contain variational parameters. All adjustable parameters in the Jastrow factor and backflow terms were optimized by minimizing the variance as well as the variational energy at the VMC level. ${ }^{72,73}$ If not explicitly stated otherwise, all of our calculations were conducted using the SJ trial wave function including $1 \mathrm{~b}, 2 \mathrm{~b}, 3 \mathrm{~b}$, and $p$ terms augmented by the BF coordinate transformation.

Beside the usage of twist-averaged boundary conditions (TABC) to correct finite-size errors, ${ }^{74}$ we extrapolated the energy per atom to the thermodynamic limit by fitting our twist-averaged DMC results for different system sizes to $E(N)=a N^{-b}+E(\infty)$, where $a$ and $b$ are fitting parameters and $E(\infty)$ is the eventual energy per atom in the infinite-system limit. The corresponding energies per atom as a function of system size are shown in Figs. 1-4 of the supplementary material. Depending on simulation cell size, we used 8, 12, and 16 randomly chosen twists. ${ }^{75}$ The enthalpy was evaluated by differentiating the polynomial fit of our finite-size-corrected $\mathrm{DMC}$ energies as a function of volume. Our DMC calculations were performed using a time step of 0.005 a.u. Due to the fact

TABLE II. The DMC total energies of the $\mathrm{C} 2 / \mathrm{c} \mathrm{HS}_{2}$ structure at four distinct pressures. The energies are calculated for $N_{1}=24, N_{2}=96$, and $N_{3}=192$ particles in the unit cell, respectively. The extrapolated DMC energy at the infinite system size limit is denoted by $\mathrm{E}(\infty)$. All energies are in eV/atom.

\begin{tabular}{lcccc}
\hline \hline $\mathrm{P}(\mathrm{GPa})$ & $\mathrm{E}\left(N_{1}\right)$ & $\mathrm{E}\left(N_{2}\right)$ & $\mathrm{E}\left(N_{3}\right)$ & $\mathrm{E}(\infty)$ \\
\hline 150 & $-187.757(1)$ & $-187.5549(8)$ & $-187.4934(6)$ & $-187.469(2)$ \\
200 & $-187.254(1)$ & $-186.9123(8)$ & $-186.8828(5)$ & $-186.817(2)$ \\
250 & $-186.767(1)$ & $-186.3066(7)$ & $-186.2809(6)$ & $-186.187(2)$ \\
300 & $-186.348(1)$ & $-185.7316(7)$ & $-185.6965(5)$ & $-185.572(2)$ \\
\hline \hline
\end{tabular}


TABLE III. Total energies of the C $2 / \mathrm{m}$ phase of $\mathrm{HS}$ at the DMC level of theory for four different pressures. The energies are given in $\mathrm{eV} /$ atom and are calculated for $N_{1}=64, N_{2}=128$, and $N_{3}=256$ particles in the unit cell, respectively. The extrapolated DMC energy at the infinite system size limit is denoted by $\mathrm{E}(\infty)$.

\begin{tabular}{lcccc}
\hline \hline $\mathrm{P}(\mathrm{GPa})$ & $\mathrm{E}\left(N_{1}\right)$ & $\mathrm{E}\left(N_{2}\right)$ & $\mathrm{E}\left(N_{3}\right)$ & $\mathrm{E}(\infty)$ \\
\hline 150 & $-144.6139(8)$ & $-144.5199(5)$ & $-144.4455(4)$ & $-144.398(1)$ \\
200 & $-143.9814(7)$ & $-143.8912(6)$ & $-143.8268(5)$ & $-143.781(1)$ \\
250 & $-143.2503(8)$ & $-143.1232(6)$ & $-143.0780(5)$ & $-143.014(1)$ \\
300 & $-142.8490(7)$ & $-142.6977(6)$ & $-142.6069(5)$ & $-142.531(1)$ \\
\hline \hline
\end{tabular}

that there is a strong tendency for the time step error to cancel between the different phases, the resulting bias in the relative free energies is insignificant.

\section{RESULTS AND DISCUSSION}

\section{A. DMC total energies}

In the following, we are revisiting the crystal structures of Ref. 35. Specifically, we begin with investigating the monoclinic $\mathrm{C} 2 / \mathrm{c}$ and $\mathrm{C} 2 / \mathrm{m}$ structures of $\mathrm{HS}_{2}$. The resulting total energies as a function of pressure at the DMC level of theory for the two $\mathrm{HS}_{2}$ structures at different system sizes and the extrapolation to the thermodynamic limit are shown in Tables I and II, respectively. Comparing the DMC results for the $\mathrm{C} 2 / \mathrm{m}$ and $\mathrm{C} 2 / \mathrm{c}$ structures of $\mathrm{HS}_{2}$, we find that at the same pressure, the total energy of the $\mathrm{C} 2 / \mathrm{c}$ phase is throughout lower than that of the $\mathrm{C} 2 / \mathrm{m}$ structure. Starting from $150 \mathrm{GPa}$, the difference is as large as $299.7 \mathrm{meV} /$ atom, but is strictly decreasing to $173 \mathrm{meV} /$ atom for $300 \mathrm{GPa}$.

The DMC total energies for the $\mathrm{C} 2 / \mathrm{m}$ and $\mathrm{I}_{1} /$ amd structures of HS are shown in Tables III and IV, respectively. As before, all energies are calculated for different number of particles in the unit cell and extrapolated to the thermodynamic limit. Even though the difference in varying, the $\mathrm{C} 2 / \mathrm{m}$ structure of HS is energetically throughout lower by about $500 \mathrm{meV} / \mathrm{atom}$ than the corresponding $\mathrm{I}_{1} / \mathrm{amd}$ phase. More precisely, at a pressure of $150,200,250$, and $300 \mathrm{GPa}$, the differences between the two structures are 580, 634, 479, and $582 \mathrm{meV} /$ atom, respectively.

Among the various potential products of the decomposition of hydrogen sulphide, $\mathrm{H}_{3} \mathrm{~S}$ is a particular intriguing candidate for conventional, but high-temperature BCS superconductivity. Interestingly, recent DFT-based crystal structure prediction simulations suggested that at high-pressure, $\mathrm{H}_{3} \mathrm{~S}$ in its trigonal R3m and cubic Im-3m structures are the most

TABLE IV. The DMC total energies of the $\mathrm{I}_{1} / \mathrm{amd}$ HS structure at four distinct pressures. The energies are calculated for $N_{1}=32, N_{2}=64$, and $N_{3}=128$ particles in the unit cell, respectively. The extrapolated DMC energy at the infinite system size limit is denoted by $\mathrm{E}(\infty)$. All energies are in eV/atom.

\begin{tabular}{lcccc}
\hline \hline $\mathrm{P}(\mathrm{GPa})$ & $\mathrm{E}\left(N_{1}\right)$ & $\mathrm{E}\left(N_{2}\right)$ & $\mathrm{E}\left(N_{3}\right)$ & $\mathrm{E}(\infty)$ \\
\hline 150 & $-145.251(1)$ & $-144.5755(8)$ & $-144.1563(5)$ & $-143.819(2)$ \\
200 & $-144.988(1)$ & $-144.1049(9)$ & $-143.5889(5)$ & $-143.147(2)$ \\
250 & $-144.671(1)$ & $-143.6350(9)$ & $-143.0537(6)$ & $-142.535(2)$ \\
300 & $-144.332(1)$ & $-143.1536(9)$ & $-142.5379(5)$ & $-141.948(2)$ \\
\hline \hline
\end{tabular}

TABLE V. Total energies of the R3m phase of $\mathrm{H}_{3} \mathrm{~S}$ at the DMC level of theory for four different pressures. The energies are given in eV/atom and are calculated for $N_{1}=48, N_{2}=96$, and $N_{3}=192$ particles in the unit cell, respectively. The extrapolated DMC energy at the infinite system size limit is denoted by $\mathrm{E}(\infty)$.

\begin{tabular}{lcccc}
\hline \hline $\mathrm{P}(\mathrm{GPa})$ & $\mathrm{E}\left(N_{1}\right)$ & $\mathrm{E}\left(N_{2}\right)$ & $\mathrm{E}\left(N_{3}\right)$ & $\mathrm{E}(\infty)$ \\
\hline 150 & $-80.0626(6)$ & $-79.8131(5)$ & $-79.7489(3)$ & $-79.624(1)$ \\
200 & $-79.7696(6)$ & $-79.5045(5)$ & $-79.4268(3)$ & $-79.294(1)$ \\
250 & $-79.4855(7)$ & $-79.2093(4)$ & $-79.1287(3)$ & $-78.991(1)$ \\
300 & $-79.1996(7)$ & $-78.9137(5)$ & $-78.8325(3)$ & $-78.689(1)$ \\
\hline \hline
\end{tabular}

likely products of the decomposition of $\mathrm{H}_{2} \mathrm{~S},{ }^{22}$ which is why they are also revisited here by means of DMC calculations. The corresponding total energies, as computed by DMC, are listed in Tables V and VI, respectively. We find that in thermodynamic limit, the Im-3m structure is energetically more favorable than the $\mathrm{R} 3 \mathrm{~m}$ phase of $\mathrm{H}_{3} \mathrm{~S}$ over the whole pressure range considered here.

\section{B. Backflow wavefunction}

However, due to the necessary fixed-node approximation in order to cope with the infamous fermion sign-problem, ${ }^{76,77}$ the fixed-node DMC method samples the variationally optimal many-electron wave function, which is consistent with an $a$ priori given nodal surface of a presumed trial wave function, instead of the exact ground-state wave function. ${ }^{50}$ [The nodal surface of an $N$-electron wave function $\Psi\left(\boldsymbol{r}_{1}, \boldsymbol{r}_{2}, \ldots, \boldsymbol{r}_{N}\right)$ is the $(3 N-1)$-dimensional hypersurface on which $\Psi$ is zero.] Therefore, the accuracy of the presumed trial wave function critically determines the quality of the eventual results via the nodal surface, which represents the sole approximation of the employed fixed-node DMC method.

As already alluded to above, using the so-called BF coordinate transformation, ${ }^{78-80}$ the orbitals in the Slater determinant are evaluated not at the actual electron positions, but on quasi-electron positions that are functions of all the particle coordinates. However, the BF function, which describes the offset of the quasi-electron coordinates relative to the actual coordinates, contains free parameters, which are determined by a variational optimization of the trial wave function. In this way, the nodes of the BF trial wave function are no longer fixed, but do have some flexibility to move during the trial wave function optimization in order to further minimize the variational energy.

Here, we have employed a large variety of different trial wave functions within our VMC and DMC energies of

TABLE VI. The DMC total energies of the Im-3m $\mathrm{H}_{3} \mathrm{~S}$ structure at four distinct pressures. The energies are calculated for $N_{1}=64, N_{2}=128$, and $N_{3}=256$ particles in the unit cell, respectively. The extrapolated DMC energy at the infinite system size limit is denoted by $\mathrm{E}(\infty)$. All energies are in eV/atom.

\begin{tabular}{lcccc}
\hline \hline $\mathrm{P}(\mathrm{GPa})$ & $\mathrm{E}\left(N_{1}\right)$ & $\mathrm{E}\left(N_{2}\right)$ & $\mathrm{E}\left(N_{3}\right)$ & $\mathrm{E}(\infty)$ \\
\hline 150 & $-79.3944(5)$ & $-79.6212(4)$ & $-79.6717(3)$ & $-79.785(1)$ \\
200 & $-79.0955(5)$ & $-79.3326(4)$ & $-79.3767(3)$ & $-79.495(1)$ \\
250 & $-78.7941(5)$ & $-79.0420(4)$ & $-79.0851(3)$ & $-79.209(1)$ \\
300 & $-78.4924(4)$ & $-78.7555(4)$ & $-78.7934(3)$ & $-78.925(1)$ \\
\hline \hline
\end{tabular}


TABLE VII. The VMC and DMC total energies for the $\mathrm{C} 2 / \mathrm{m}$ phase of $\mathrm{HS}_{2}$ as calculated using the $\mathrm{SJ}(1 \mathrm{~b}+2 \mathrm{~b}), \mathrm{SJ}(1 \mathrm{~b}+2 \mathrm{~b}+p), \mathrm{SJ}(1 \mathrm{~b}+2 \mathrm{~b}+3 \mathrm{~b})$, $\mathrm{SJ}(1 \mathrm{~b}+2 \mathrm{~b}+3 \mathrm{~b}+p)$, and $\mathrm{BSJ}(1 \mathrm{~b}+2 \mathrm{~b})$ and $\mathrm{BSJ}(1 \mathrm{~b}+2 \mathrm{~b}+3 \mathrm{~b}+p)$ trial wave functions. All energies are in Hartree per primitive unit cell.

\begin{tabular}{lccc}
\hline \hline $\mathrm{WF}$ & VMC & DMC & variance \\
\hline $\mathrm{SJ}(1 \mathrm{~b}+2 \mathrm{~b})$ & $-81.9882(5)$ & $-82.1819(5)$ & $6.97(2)$ \\
$\mathrm{SJ}(1 \mathrm{~b}+2 \mathrm{~b}+p)$ & $-82.0508(3)$ & $-82.1848(5)$ & $6.22(4)$ \\
$\mathrm{SJ}(1 \mathrm{~b}+2 \mathrm{~b}+3 \mathrm{~b})$ & $-82.0150(4)$ & $-82.1886(5)$ & $6.54(3)$ \\
$\mathrm{SJ}(1 \mathrm{~b}+2 \mathrm{~b}+3 \mathrm{~b}+p)$ & $-82.0772(3)$ & $-82.1932(4)$ & $5.82(2)$ \\
$\mathrm{BSJ}(1 \mathrm{~b}+2 \mathrm{~b})$ & $-82.0806(3)$ & $-82.2259(4)$ & $5.10(3)$ \\
$\mathrm{BSJ}(1 \mathrm{~b}+2 \mathrm{~b}+3 \mathrm{~b}+p)$ & $-82.1683(3)$ & $-82.2423(4)$ & $4.17(4)$ \\
\hline \hline
\end{tabular}

$\mathrm{HS}_{2}$ and $\mathrm{H}_{3} \mathrm{~S}$, which are the systems with of lowest and highest hydrogen densities we have considered. Specifically, we applied SJ-type trial wave functions including one- and two-body terms $(\mathrm{SJ}(1 \mathrm{~b}+2 \mathrm{~b}))$, an additional three-body term $(\mathrm{SJ}(1 \mathrm{~b}+2 \mathrm{~b}+3 \mathrm{~b}))$, as well as the respective versions that are augmented by a $p$-term denoted as $\mathrm{SJ}(1 \mathrm{~b}+2 \mathrm{~b}+p)$ and $\mathrm{SJ}(1 \mathrm{~b}+2 \mathrm{~b}+3 \mathrm{~b}+p)$, respectively. In addition, BackflowSlater-Jastrow (BSJ) trial wave functions including oneand two-body terms $(B S J(1 b+2 b))$ and the subsequent variant including an additional three-body and the $\mathrm{p}$ term $(\operatorname{BSJ}(1 b+2 b+3 b+p))$ are considered. The resulting VMC and DMC total energies for the $\mathrm{C} 2 / \mathrm{m}$ phase of $\mathrm{HS}_{2}$ and Im-3m $\mathrm{H}_{3} \mathrm{~S}$ structure are listed in Tables VII and VIII, respectively. The energy gain of the various trial wave functions with respect to the $\mathrm{SJ}(1+2 \mathrm{~b})$ approach using the VMD and DMC methods for the two different systems are shown in Fig. 1. It is apparent that the addition of the three-body term and more so the $p$ term substantially reduces the VMC energy, but the DMC is only marginally affected since the initial nodal structure is identical. The usage of the BF transformation, however, improves the nodal surface and hence entails an energy lowering at the VMC and the DMC levels. Interestingly, the gain in energy for $\mathrm{HS}_{2}$ is much more pronounced than for $\mathrm{H}_{3} \mathrm{~S}$, which demonstrates the importance of an accurate trial wave function for the former. For the Im-3m phase of $\mathrm{H}_{3} \mathrm{~S}$ the energy gain is -1.634 and $-0.56 \mathrm{eV}$ at the VMC and DMC levels of theory, while for the $\mathrm{C} 2 / \mathrm{m} \mathrm{HS}_{2}$ structure, the energy gain can be as high as -4.898 and $-1.643 \mathrm{eV}$, respectively.

The increased accuracy of the BF wave function, however, comes at a rather high computational cost, which is due to the necessity to evaluate the orbitals and their first two derivatives and moreover also the collective BF coordinates, because every element of the Slater matrix must be

TABLE VIII. The VMC and DMC total energies of the Im- $3 \mathrm{~m} \mathrm{H}_{3} \mathrm{~S}$ structussre as calculated using the $\mathrm{SJ}(1 \mathrm{~b}+2 \mathrm{~b}), \mathrm{SJ}(1 \mathrm{~b}+2 \mathrm{~b}+p), \mathrm{SJ}(1 \mathrm{~b}+2 \mathrm{~b}+3 \mathrm{~b})$, $\mathrm{SJ}(1 \mathrm{~b}+2 \mathrm{~b}+3 \mathrm{~b}+p)$, and $\mathrm{BSJ}(1 \mathrm{~b}+2 \mathrm{~b})$ and $\mathrm{BSJ}(1 \mathrm{~b}+2 \mathrm{~b}+3 \mathrm{~b}+p)$ trial wave functions. All energies are in Hartree per primitive unit cell.

\begin{tabular}{lccc}
\hline \hline WF & VMC & DMC & variance \\
\hline $\mathrm{SJ}(1 \mathrm{~b}+2 \mathrm{~b})$ & $-23.1044(3)$ & $-23.1650(2)$ & $4.21(4)$ \\
$\mathrm{SJ}(1 \mathrm{~b}+2 \mathrm{~b}+p)$ & $-23.1223(2)$ & $-23.1663(2)$ & $3.75(1)$ \\
$\mathrm{SJ}(1 \mathrm{~b}+2 \mathrm{~b}+3 \mathrm{~b})$ & $-23.1134(2)$ & $-23.1674(3)$ & $3.90(2)$ \\
$\mathrm{SJ}(1 \mathrm{~b}+2 \mathrm{~b}+3 \mathrm{~b}+p)$ & $-23.1307(2)$ & $-23.1680(2)$ & $3.54(1)$ \\
$\operatorname{BSJ}(1 \mathrm{~b}+2 \mathrm{~b})$ & $-23.1381(2)$ & $-23.1803(3)$ & $2.81(2)$ \\
$\operatorname{BSJ}(1 \mathrm{~b}+2 \mathrm{~b}+3 \mathrm{~b}+p)$ & $-23.1645(2)$ & $-23.1856(1)$ & $2.278(9)$ \\
\hline \hline
\end{tabular}

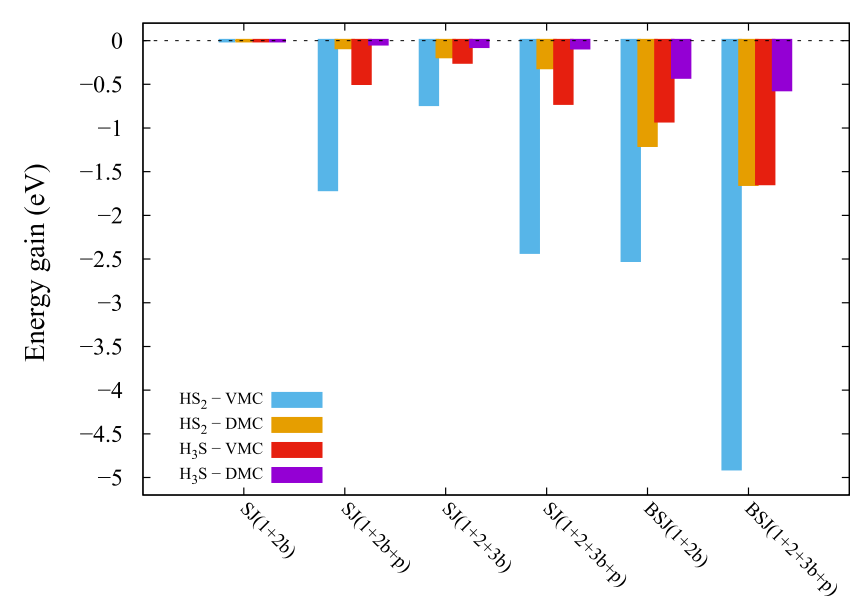

FIG. 1. The reduction of variational energy for the $\mathrm{HS}_{2}$ and $\mathrm{H}_{3} \mathrm{~S}$ systems using different trial wave functions with respect to the $\mathrm{SJ}(1 \mathrm{~b}+2 \mathrm{~b})$ approach using the VMC and DMC methods.

updated every time a single electron is moved. Even though this is partially compensated by the fact that the less complex BSJ $(1 b+2 b)$ trial wave function is equally accurate than the much more complex SJ $(1 \mathrm{~b}+2 \mathrm{~b}+3 \mathrm{~b}+p)$ calculations, we have elected to use the former instead of the latter in the following DMC calculations of the enthalpy-pressure phase diagram calculations.

\section{DMC enthalpy-pressure phase diagram}

In order to compute the enthalpy-pressure phase diagrams for the different structures, we fitted our extrapolated DMC total energies as a function of volume $V$ against a model equations of state $E(V)$. We found that a quadratic polynomial is a sufficiently accurate representation of our actual DMC energies. Using this model, it is straightforward to calculate the pressure $P(V)=-d E(V) / d V$ as a function of $V$ and thus also the enthalpy per atom $H=E+P V$.

In previous DFT calculations including ZPE correction, it was predicted that at $200 \mathrm{GPa} 5 \mathrm{H}_{2} \mathrm{~S}$ decomposes into $3 \mathrm{H}_{3} \mathrm{~S}$ and $\mathrm{HS}_{2}$, where $\mathrm{HS}_{2}$ adopts $\mathrm{C} 2 / \mathrm{c}$ symmetry, but undergoes a phase

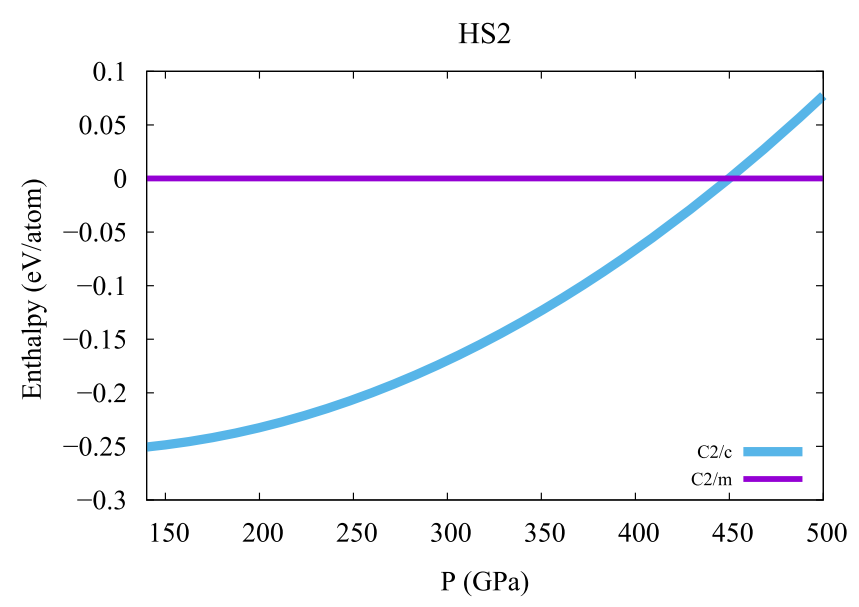

FIG. 2. The DMC enthalpy as a function of pressure of the $\mathrm{C} 2 / \mathrm{c} \mathrm{HS}_{2}$ structure relative to the $\mathrm{C} 2 / \mathrm{c}$ phase. The estimated uncertainties in the DMC enthalpies due to statistical and systematic errors are represented by the widths of the corresponding lines. 
HS

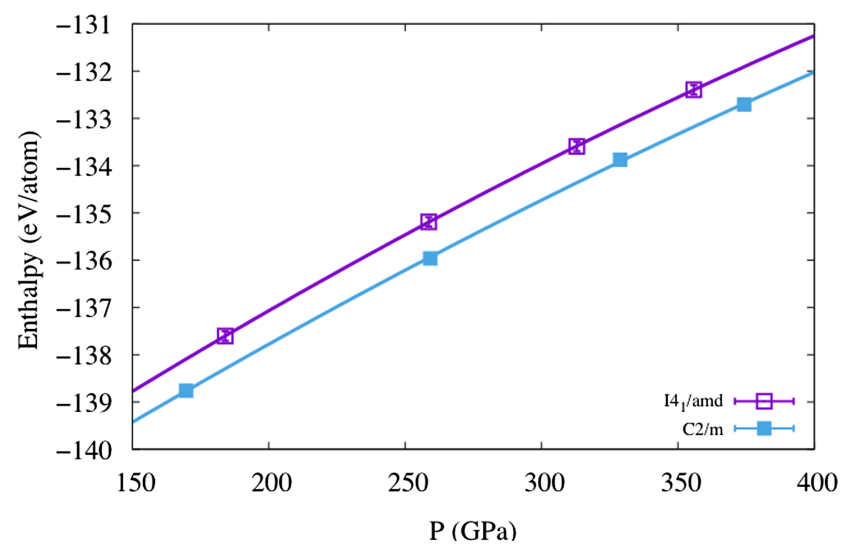

FIG. 3. The DMC enthalpy of the $\mathrm{C} 2 / \mathrm{m}$ and $\mathrm{I} 4_{1} / \mathrm{amd}$ phases of HS as a function of pressure. The corresponding error bars are smaller than the size of the data points.

transition to the more stable $\mathrm{C} 2 / \mathrm{m}$ structure at $250 \mathrm{GPa} .{ }^{35} \mathrm{How}-$ ever, the present DMC enthalpies indicate that the $\mathrm{C} 2 / \mathrm{c} \mathrm{HS}_{2}$ structure is more stable than the $\mathrm{C} 2 / \mathrm{m}$ phase up to $440 \mathrm{GPa}$, as it is shown in Fig. 2. In fact, the enthalpy difference between the $\mathrm{C} 2 / \mathrm{c}$ and $\mathrm{C} 2 / \mathrm{m}$ phases of $\mathrm{HS}_{2}$ is much larger than the ZPE correction as estimated by DFT. ${ }^{35}$ Moreover, DFT-based crystal structure searches predict that the energetically most favorable phase of $\mathrm{HS}$ at $200 \mathrm{GPa}$ is $\mathrm{I}_{1} /$ amd and at $300 \mathrm{GPa}$ $\mathrm{C} 2 / \mathrm{m}^{35}$ The present DMC enthalpy-pressure curves of HS are shown in Fig. 3. At variance to DFT, they suggest that the $\mathrm{C} 2 / \mathrm{m}$ phase of $\mathrm{HS}$ is more stable than the $\mathrm{I} 4_{1} /$ amd HS structure over the whole pressure range from 150 to $400 \mathrm{GPa}$. In fact, the difference in enthalpy between the $\mathrm{C} 2 / \mathrm{m}$ and $\mathrm{I} 4_{1} /$ amd phases is more than $600 \mathrm{meV} /$ atom and is even larger with increasing pressure. Previous crystal structure prediction calculations using DFT indicate that the $\mathrm{R} 3 \mathrm{~m} \mathrm{H}_{3} \mathrm{~S}$ structure is stable at $130 \mathrm{GPa}$ and that the $\mathrm{Im}-3 \mathrm{~m}$ phase of $\mathrm{H}_{3} \mathrm{~S}$ is the most likely candidate at pressures larger than $200 \mathrm{GPa}$. Our DMC enthalpy-pressure phase diagram for $\mathrm{H}_{3} \mathrm{~S}$ system is illustrated in Fig. 4.

Our DFT-PBE results indicate that the enthalpy difference between the $\mathrm{Im}-3 \mathrm{~m}$ and $\mathrm{R} 3 \mathrm{~m}$ structures of $\mathrm{H}_{3} \mathrm{~S}$ is less than $3 \mathrm{meV} / \mathrm{atom}$ in the studied pressure range. This small

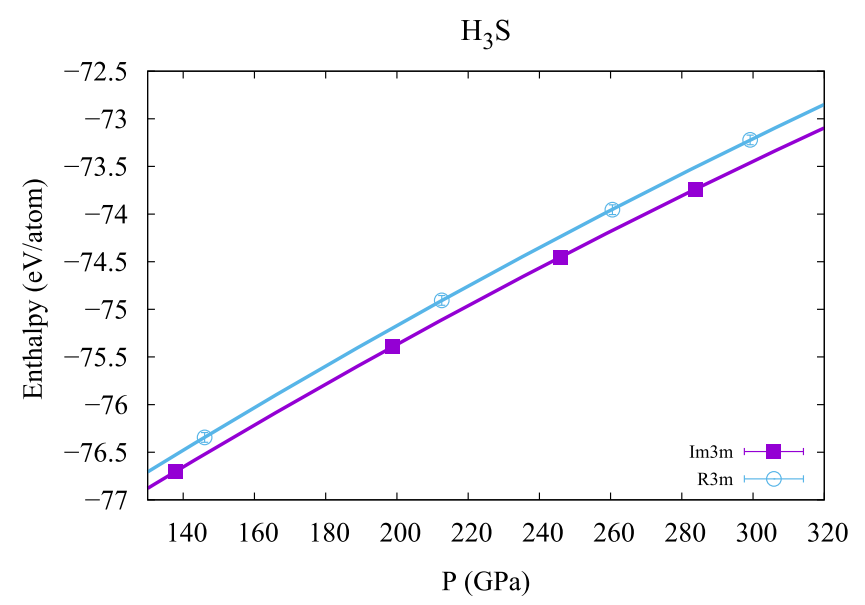

FIG. 4. The DMC enthalpy of the Im-3m and $\mathrm{R} 3 \mathrm{~m}$ phases of $\mathrm{H}_{3} \mathrm{~S}$ as a function of pressure. The corresponding error bars are smaller than the size of the data points. energy difference agrees with previous DFT-PBE calculations where it is stated that the Im-3m and R3m structures are almost identical in energy at pressures above $175 \mathrm{GPa}^{22,32}$ Determining the corresponding enthalpy-pressure phase diagram on such a tiny energy scale based on stochastic DMC simulations is a very daunting task and computationally tremendously demanding. We estimate that the statistical and systematic errors in our DMC enthalpy simulations are larger than the DFT-PBE energy difference between the Im-3m and R3m phases. Although, our DMC enthalpy-pressure calculations predict that the $\mathrm{Im}-3 \mathrm{~m} \mathrm{H}_{3} \mathrm{~S}$ structure is enthalpically more favorable up to pressures of at least $320 \mathrm{GPa}$, which is in agreement with very recent experimental results, ${ }^{29}$ this prediction needs further investigation.

\section{CONCLUSION}

In summary, our DMC results lead to a revision of the enthalpy-pressure phase diagram between 150 and 320 GPa. Specifically, we find that the $C 2 / c \mathrm{HS}_{2}, C 2 / m \mathrm{HS}$ and Im $-3 m \mathrm{H}_{3} \mathrm{~S}$ structures are energetically most favorable within the considered pressure range. However, it is well known that the ZPE corrections play an important role in the numerical determination of the phase diagram hydrogen-rich systems. ${ }^{5,47,61}$ Nevertheless, DFT calculations of others have shown that the energy difference between $\mathrm{H}_{2} \mathrm{~S}$ and $\mathrm{S}+\mathrm{H}_{2}$ due to $\mathrm{ZPE}$ is about $6 \mathrm{meV} / \mathrm{f}$.u. at $160 \mathrm{GPa},{ }^{41}$ which is much smaller than our DMC enthalpy differences. We thus predict that the effect of ZPE on our DMC results is negligible. We conclude by noting that the present DMC results are compatible with very recent DFT results including $\mathrm{ZPE},{ }^{38}$ so that in the most important case of $\mathrm{H}_{3} \mathrm{~S}$, two state of the art calculations at rather different levels of theories provide similar results that are in agreement with experiment.

\section{SUPPLEMENTARY MATERIAL}

In the supplementary material, we provide the details of the fitting of $E(N)$ and $E(V)$, where $E$ is the DMC total energy, $N$ the number of atoms in the unit cell, and $V$ the corresponding volume. We fitted the twist-averaged DMC results for three system sizes to $E(N)=a N^{-b}+E(\infty)$, where $a$ and $b$ are fitting parameters, while $E(\infty)$ is the energy per atom in the infinitesystem limit.

\section{ACKNOWLEDGMENTS}

This work made use of computing facilities provided by ARCHER, the UK national super computing service, and by the High Performance Computing Service of Imperial College London, as well as of the OCuLUS system of the Paderborn Center for Parallel Computing $\left(\mathrm{PC}^{2}\right)$. In particular, we acknowledge that the results of this research have been achieved using the PRACE-3IP project (FP7 RI-312763) resource ARCHER based in UK. Support from the Thomas Young Centre under Grant Nos. TYC-101 and EP/N50869X/1 is also thankfully acknowledged.

\footnotetext{
${ }^{1}$ J. Bardeen, L. N. Cooper, and J. R. Schrieffer, Phys. Rev. 108, 1175 (1957).

${ }^{2}$ E. Wigner and H. B. Huntington, J. Chem. Phys. 3, 764 (1935).

${ }^{3}$ N. W. Ashcroft, Phys. Rev. Lett. 21, 1748 (1968).
} 
${ }^{4}$ S. Azadi and T. D. Kühne, JETP Lett. 95, 449 (2012).

${ }^{5}$ S. Azadi, W. M. C. Foulkes, and T. D. Kühne, New J. Phys. 15, 113005 (2013).

${ }^{6}$ W. J. Nellis, S. T. Weir, and A. C. Mitchell, Science 273, 936 (1996).

${ }^{7}$ S. T. Weir, W. J. Nellis, and A. C. Mitchell, Phys. Rev. Lett. 76, 1860 (1996).

${ }^{8}$ W. J. Nellis, S. T. Weir, and A. C. Mitchell, Phys. Rev. B 59, 3434 (1999).

${ }^{9}$ M. I. Eremets and I. A. Troyan, Nat. Mater. 10, 927 (2011).

${ }^{10}$ M. Zaghoo, A. Salamat, and I. F. Silvera, Phys. Rev. B 93, 155128 (2016).

${ }^{11}$ N. W. Ashcroft, Phys. Rev. Lett. 92, 187002 (2004).

${ }^{12} \mathrm{~J}$. Feng, W. Grochala, T. Jaron, R. Hoffmann, A. Bergara, and N. W. Ashcroft, Phys. Rev. Lett. 96, 017006 (2006).

${ }^{13}$ T. A. Strobel, P. Ganesh, M. Somayazulu, P. R. C. Kent, and R. J. Hemley, Phys. Rev. Lett. 107, 255503 (2011).

${ }^{14}$ M. I. Eremets, I. A. Trojan, S. A. Medvedev, J. S. Tse, and Y. Yao, Science 319, 1506 (2008).

${ }^{15}$ D. Y. Kim, R. H. Scheicher, C. J. Pickard, R. J. Needs, and R. Ahuja, Phys. Rev. Lett. 107, 117002 (2011).

${ }^{16}$ T. Scheler, O. Degtyareva, M. Marques, C. L. Guillaume, J. E. Proctor, S. Evans, and E. Gregoryanz, Phys. Rev. B 83, 214106 (2011).

${ }^{17}$ X. F. Zhou, A. R. Oganov, X. Dong, L. Zhang, Y. Tian, and H. T. Wang, Phys. Rev. B 84, 054543 (2011).

${ }^{18}$ D. Y. Kim, R. H. Scheicher, H. k. Mao, T. W. Kang, and R. Ahuja, Proc. Natl. Acad. Sci. U. S. A. 107, 2793 (2010).

${ }^{19}$ G. Gao, A. R. Oganov, P. Li, Z. Li, H. Wang, T. Cui, Y. Ma, A. Bergara, A. O. Lyakhov, T. Iitaka, and G. Zou, Proc. Natl. Acad. Sci. U. S. A. 107, 1317 (2010)

${ }^{20}$ G. Gao, A. R. Oganov, A. Bergara, M. Martinez-Canales, T. Cui, T. Iitaka, Y. Ma, and G. Zou, Phys. Rev. Lett. 101, 107002 (2008).

${ }^{21}$ Y. Li, J. Hao, H. Liu, Y. Li, and Y. Ma, J. Chem. Phys. 140, 174712 (2014).

${ }^{22}$ D. Duan, Y. Liu, F. Tian, D. Li, X. Huang, Z. Zhao, H. Yu, B. Liu, W. Tian, and T. Cui, Sci. Rep. 4, 6968 (2014).

${ }^{23}$ A. P. Drozdov, M. I. Eremets, I. A. Troyan, V. Ksenofontov, and S. I. Shylin, Nature 525, 73 (2015).

${ }^{24}$ I. A. Troyan, A. Gavriliuk, R. Rüffer, A. Chumakov, A. Mironovich, I. Lyubutin, D. Perekalin, A. P. Drozdov, and M. I. Eremets, Science 351, 1303 (2016).

${ }^{25}$ J. G. Bednorz and K. A. Mueller, Z. Phys. B 64, 189 (1986).

${ }^{26}$ A. Schilling, M. Cantoni, J. D. Guo, and H. R. Ott, Nature 363, 56 (1993).

${ }^{27}$ L. Gao, Y. Y. Xue, F. Chen, Q. Xiong, R. L. Meng, D. Ramirez, C. W. Chu, J. H. Eggert, and H. K. Mao, Phys. Rev. B 50, 4260(R) (1994).

${ }^{28}$ H. Luo, R. G. Greene, and A. L. Ruoff, Phys. Rev. Lett. 71, 2943 (1993).

${ }^{29}$ M. Einaga, M. Sakata, T. Ishikawa, K. Shimizu, M. I. Eremets, A. P. Drozdov, I. A. Troyan, N. Hirao, and Y. Ohishi, Nat. Phys. 12, 835 (2016).

${ }^{30}$ N. Bernstein, C. Stephen Hellberg, M. D. Johannes, I. I. Mazin, and M. J. Mehl, Phys. Rev. B 91, 060511(R) (2015).

${ }^{31}$ R. Akashi, M. Kawamura, S. Tsuneyuki, Y. Nomura, and R. Arita, Phys. Rev. B 91, 224513 (2015).

${ }^{32}$ J. A. Flores-Livas and E. K. U. Gross, Eur. Phys. J. B 89, 63 (2016).

${ }^{33}$ A. F. Goncharov, S. S. Lobanov, I. Kruglov, X.-M. Zhao, X.-J. Chen, A. R. Oganov, Z. Konôpková, and V. B. Prakapenka, Phys. Rev. B 93, 174105 (2016).

${ }^{34}$ B. Guigue, A. Marizy, and P. Loubeyre, Phys. Rev. B 95, 020104(R) (2017).

${ }^{35}$ I. Errea, M. Calandra, C. J. Pickard, J. Nelson, R. J. Needs, Y. Li, H. Liu, Y. Zhang, Y. Ma, and F. Mauri, Phys. Rev. Lett. 114, 157004 (2015).

${ }^{36}$ D. A. Papaconstantopoulos, B. M. Klein, M. J. Mehl, and W. E. Pickett, Phys. Rev. B 91, 184511 (2015).

${ }^{37}$ Y. Li, L. Wang, H. Liu, Y. Zhang, J. Hao, C. J. Pickard, J. R. Nelson, R. J. Needs, W. Li, Y. Huang, I. Errea, M. Calandra, F. Mauri, and Y. Ma, Phys. Rev. B 93, 020103(R) (2016).

${ }^{38}$ I. Errea, M. Calandra, C. J. Pickard, J. R. Nelson, R. J. Needs, Y. Li, H. Liu, Y. Zhang, Y. Ma, and F. Mauri, Nature 532, 81 (2016).

${ }^{39}$ J. P. Perdew, K. Burke, and M. Ernzerhof, Phys. Rev. Lett. 77, 3865 (1996).

${ }^{40}$ I. Errea, M. Calandra, and F. Mauri, Phys. Rev. Lett. 111, 177002 (2013).
${ }^{41}$ S. Azadi, N. D. Drummond, and W. M. C. Foulkes, Phys. Rev. B 95, 035142 (2017).

${ }^{42}$ C. J. Pickard and R. J. Needs, Nat. Phys. 3, 473 (2007).

${ }^{43}$ D. Duan, X. Huang, F. Tian, D. Li, H. Yu, Y. Liu, Y. Ma, B. Liu, and T. Cui, Phys. Rev. B. 91, 180502(R) (2015).

${ }^{44}$ X.-J. Chen, J.-L. Wang, V. V. Struzhkin, H.-k. Mao, R. J. Hemley, and H.-Q. Lin, Phys. Rev. Lett. 101, 077002 (2008).

${ }^{45}$ M. Martinez-Canales, A. R. Oganov, Y. Ma, Y. Yan, A. O. Lyakhov, and A. Bergara, Phys. Rev. Lett. 102, 087005 (2009).

${ }^{46}$ R. Singh, S. Azadi, and T. D. Kühne, Phys. Rev. B 90, 014110 (2014).

${ }^{47}$ S. Azadi and W. M. C. Foulkes, Phys. Rev. B. 88, 014115 (2013).

${ }^{48}$ M. A. Morales, J. M. McMahon, C. Pierleoni, and D. M. Ceperley, Phys. Rev. B 87, 184107 (2013).

${ }^{49}$ R. C. Clay, J. Mcminis, J. M. McMahon, C. Pierleoni, D. M. Ceperley, and M. A. Morales, Phys. Rev. B 89, 184106 (2014).

${ }^{50}$ D. M. Ceperley and B. J. Alder, Phys. Rev. Lett. 45, 566 (1980).

${ }^{51}$ W. M. C. Foulkes, L. Mitas, R. J. Needs, and G. Rajagopal, Rev. Mod. Phys. 73, 33 (2001).

${ }^{52}$ M. Marchi, S. Azadi, M. Casula, and S. Sorella, J. Chem. Phys. 131, 154116 (2009).

${ }^{53}$ M. D. Brown, J. R. Trail, P. López Ríos, and R. J. Needs, J. Chem. Phys. 126, 224110 (2007).

${ }^{54}$ J. R. Trail and R. L. Needs, J. Chem. Phys. 128, 204103 (2008).

${ }^{55}$ S. Azadi, R. Singh, and T. D. Kühne, Int. J. Quantum Chem. 115, 1673 (2015).

${ }^{56}$ S. Azadi and R. E. Cohen, J. Chem. Phys. 143, 104301 (2015).

${ }^{57}$ M. Marchi, S. Azadi, and S. Sorella, Phys. Rev. Lett. 107, 086807 (2011).

${ }^{58}$ E. Mostaani, N. D. Drummond, and V. I. Falḱo, Phys. Rev. Lett. 115, 115501 (2015).

${ }^{59}$ S. Azadi and W. M. C. Foulkes, J. Chem. Phys. 143, 102807 (2015).

${ }^{60}$ N. D. Drummond, B. Monserrat, J. H. Lloyd-Williams, P. Lòpes Rìos, and R. J. Needs, Nat. Commun. 6, 7749 (2015).

${ }^{61}$ S. Azadi, B. Monserrat, W. M. C. Foulkes, and R. J. Needs, Phys. Rev. Lett. 112, 165501 (2014).

${ }^{62}$ J. M. McMahon, M. A. Morales, C. Pierleoni, and D. M. Ceperley, Rev. Mod. Phys. 84, 1607 (2012).

${ }^{63}$ S. J. Clark, M. D. Segall, C. J. Pickard, P. J. Hasnip, M. J. Probert, K. Refson, and M. C. Payne, Z. Kristallogr. 220, 567 (2005).

${ }^{64}$ D. Vanderbilt, Phys. Rev. B 41, 7892 (1990).

${ }^{65}$ R. J. Needs, M. D. Towler, N. D. Drummond, and P. López Ríos, J. Phys.: Condens. Matter 22, 023201 (2010).

${ }^{66}$ J. R. Trail and R. J. Needs, J. Chem. Phys. 122, 174109 (2005).

${ }^{67}$ J. R. Trail and R. J. Needs, J. Chem. Phys. 122, 014112 (2005).

${ }^{68}$ S. Azadi, C. Cavazzoni, and S. Sorella, Phys. Rev. B 82, 125112 (2010).

${ }^{69}$ D. Alfè and M. J. Gillan, Phys. Rev. B 70, 161101 (2004).

${ }^{70}$ N. D. Drummond, M. D. Towler, and R. J. Needs, Phys. Rev. B 70, 235119 (2004).

${ }^{71}$ P. López Rìos, A. Ma, N. D. Drummond, M. D. Towler, and R. J. Needs, Phys. Rev. E. 74, 066701 (2006).

${ }^{72}$ C. J. Umrigar, K. G. Wilson, and J. W. Wilkins, Phys. Rev. Lett. 60, 1719 (1988).

${ }^{73}$ N. D. Drummond and R. J. Needs, Phys. Rev. B 72, 085124 (2005).

${ }^{74}$ C. Lin, F. H. Zong, and D. M. Ceperley, Phys. Rev. E 64, 016702 (2001).

${ }^{75}$ N. D. Drummond, R. J. Needs, A. Sorouri, and W. M. C. Foulkes, Phys. Rev. B 78, 125106 (2008).

${ }^{76}$ F. Calcavecchia, F. Pederiva, M. H. Kalos, and T. D. Kühne, Phys. Rev. E 90, 053304 (2014).

${ }^{77}$ F. Calcavecchia and T. D. Kühne, Eur. Phys. Lett. 110, 20011 (2015).

${ }^{78}$ R. P. Feynman and M. Cohen, Phys. Rev. 102, 1189 (1956).

${ }^{79}$ M. Holzmann, D. M. Ceperley, C. Pierleoni, and K. Esler, Phys. Rev. E 68, 046707 (2003).

${ }^{80}$ M. Taddei, M. Ruggeri, S. Moroni, and M. Holzmann, Phys. Rev. B 91, 115106 (2015). 